\title{
Evaluación de sistemas de selección en ambientes alternados e in situ en trigo Triticum aestivum $\mathrm{L}$.
}

Evaluation of selection systems in alternate and in situ environments in wheat Triticum aestivum L.

\section{Luis Fernando Campuzano D. ; José Molina G. ${ }^{2}$; Sanjaya Rajaram ${ }^{3}$}

\footnotetext{
${ }^{1}$ Ph.D. Corpoica.Villavicencio, Colombia, lcampuzano@corpoica.org.co

${ }^{2}$ Ph.D. Colegio de Posgraduados. Montecillos, México, j.dgalan@colpox.org.mx

${ }^{3}$ Ph.D. Centro Internacional de Mejoramiento de Maíz y Trigo (CIMMYT). Batán, México, srajaram@cgiar.org
}

Citar: CAMPUZANO. L.; MOLINA, J.; RAJARAM, S. 2015. Evaluación de sistemas de selección en ambientes alternados e in situ en trigo Triticum aestivum L. Rev. Cienc. Agr. 32(2):36 - 45.

Fecha de recepción: Abril 4 de 2015

Fecha de aceptación: Octubre 27 de 2015

\section{RESUMEN}

El proceso de mejoramiento en trigo alternando localidades conocido como Selección Alternada o Shuttle Breeding ha sido el camino más eficiente para introducir y seleccionar genes para resistencia a royas, insensibilidad al fotoperiodo y de enanismo; atributos de importancia para el incremento del rendimiento. Después de 35 años de su aplicación en México y en otras partes del mundo, se realizó el presente trabajo con el objetivo de evaluar las bondades del sistema alternado y determinar entre los sistemas alternados (Toluca-Cd. Obregón, Batán-Cd Obregón y Batán-Toluca) e in situ (Toluca-Toluca, Cd. Obregón-Cd. Obregón y Batán-Batán) el que proporciona el mayor número de líneas de trigo con alto rendimiento y estabilidad y la asociación de las localidades de evaluación con la adaptación especifica o general. El material genético se derivó de seis cruzamientos de trigo y después del proceso de selección masal modificada se escogieron 32 líneas por sistema, para ser evaluadas en un diseño alfa-lattice de 14 x 14 en un ciclo agrícola en tres ambientes de selección, en Ciudad Obregón, Batán y Toluca, en México. Los resultados mostraron que la selección alternada en relación con la selección in situ resultó superior en 2,2\% en rendimiento de grano y el mejor sistema fue B-T por haber presentado el mayor rendimiento y el mayor número de líneas de alto rendimiento y estables. En contraposición, los sistemas B-Y, T-T y Y-Y fueron los que aportaron el menor número de líneas estables de alto rendimiento.

Palabras clave: Estabilidad, respuesta selección, selección alternada, Triticum aestivum L. 


\begin{abstract}
Wheat breeding by alternating locations, known as Shuttle Breeding, has been the most efficient way to introduce and select genes for rust resistance, photoperiod insensitivity, and dwarfism, which are important attributes for increased yield. After 35 years of its implementation in Mexico and elsewhere in the world, this work was aimed to evaluated the benefits of the alternating system and determined which system, between alternating systems (Toluca-Cd. Obregon, Ciudad Obregon-Batán Batán -Toluca) and in situ systems (Toluca Toluca, Cd. Obregon-Cd. Obregon-Batán Batán), provides the largest number of wheat lines with high yield and stability. As well as the association with the specific or general adaptation. The genetic material was gathered from six wheat crosses and, after the modified mass selection, 32 lines were selected for each system to be evaluated on a14 x 14 alpha-lattice design, in an agricultural cycle, in three selection environments, in Cuidad Obregón, Batán, and Toluca, Mexico. The results showed that alternate selection compared to in situ site selection exhibited a 2.2\% increase in yield. BT was the best system since it showed the highest yield and greatest number of lines with high performance and stability. In contrast, BY, TT, and YY systems made the lowest contribution to the lines with highly yield and stability.
\end{abstract}

Keywords: Stability, selection response, shuttle breeding, Triticum aestivum L.

\section{INTRODUCCIÓN}

El éxito del mejoramiento genético del trigo a nivel mundial se debe en gran medida a la obtención de variedades mejoradas de alta estabilidad con resistencia a enfermedades, principalmente a royas, con alto rendimiento y calidad. Para lograr esto, se debió considerar la importancia del ambiente y la interacción genotipo $\mathrm{x}$ ambiente en la expresión de un fenotipo y la selección y estabilidad de los atributos de interés. Para ello, Borlaug (1968) desarrollo un sistema de selección denominado Shuttle Breeding (Selección alternada). Este proceso de mejoramiento en trigo alternando localidades fue el camino más eficiente para introducir y seleccionar los genes para resistencia a las royas, insensibilidad al fotoperiodo $\left(\operatorname{Ppd}_{1}\right.$ y $\left.\operatorname{Ppd}_{2}\right)$, aunado a la incorporación de los genes de enanismo $\mathrm{Rht}_{1} \mathrm{y} \mathrm{Rht}_{2}$. El resultado fue un nuevo tipo de planta, resistente al volcamiento, con un incremento significativo en el rendimiento, alta biomasa y resistencia a royas. La conjunción de todos estos factores, representó la obtención de líneas de trigo de alto grado de adaptación ecológica.
CIMMYT desarrollo germoplasma de trigos mejorados, para ser utilizados en países emergentes que siembran alrededor de 110 millones de hectáreas (Lantica et al., 2005). Para ello, se desarrolló el concepto de mega-ambiente (Rajaram et al., 1983, Rajaram y Morgounov, 1995; Reynolds et al., 2013) que se define como un área amplia, no necesariamente contigua que se presenta en más de un país $\mathrm{y}$ frecuentemente transcontinental, definido por similitudes de estreses bióticos y abióticos, requerimientos de cultivo, preferencias de consumidor y por consecuencia, por un volumen de producción.

Como evidencia del éxito del método y el uso de los megaambientes, además del reconocimiento "Revolución Verde" y el Premio Nobel para Borlaug en el año 1972, se conoce que el 58 \% del área cultivada de trigo en países desarrollados, excluyendo a China, proviene de germoplasma del CIMMYT (Byerlee y Moya, 1993; Rajaram et al., 1994) y el $70 \%$ de las variedades liberadas en países desarrollados durante el periodo 1966 - 2013 contiene germoplasma del CIMMYT en su pedigree (Byerlee y Moya, 1993; Reynolds et al., 2013). 
Lu et al. (1967) encontraron que el mayor grado de adaptación y mayor rendimiento de las líneas de soya (Glycine max L) se logró mediante la selección alternada del material segregante seleccionado en dos estaciones del año, primavera y verano. A diferencia de lo anterior, en avena (Avena sativa L.) Adegoke y Frey (1981) compararon la selección alternada con la selección in situ durante nueve generaciones en tres localidades ubicadas al norte, centro y sur del estado Iowa; demostraron que el mayor rendimiento y la estabilidad de las líneas de avena obtenidas fueron en el sistema in situ. En esta misma especie, Shabana et al. (1980) determinaron que la selección para rendimiento fue más efectiva en la selección in situ a través del ambiente de mayor productividad en comparación con el ambiente de intermedio y bajo nivel de productividad.

Quizás la experiencia de mayor trascendencia a nivel mundial con base en el impacto biológico, económico, social y político, es el sistema de selección desarrollado por Borlaug (1968) en el programa de trigo del CIMMYT en México. El número de ciclos desarrollado desde su inicio, más de 100 generaciones, la liberación de más de 1000 variedades producidas por el CIMMYT y liberadas por programas nacionales de países desarrollados y en desarrollo, han situado este sistema de selección y al programa del CIMMYT como uno de los más exitosos a nivel mundial (Trethowan et al., 2007).

El mejor reconocimiento que tiene el CIMMYT es la extrapolación de este sistema de selección a otras latitudes. Tres de los ejemplos de mayor trascendencia son los programas de Brasil, Estados Unidos y China, los cuales alternan los materiales genéticos en sus localidades y en las localidades usadas tradicionalmente por el CIMMYT en México (Reynolds y Borlaug, 2006; Reynolds et al., 2013; CIAT, 2014).
En el primer caso, los resultados han sido la combinación de las buenas características de los trigos obtenidos en México, con la tolerancia a aluminio y eficiencia en el uso del fosforo de los materiales de Brasil (Rajaram et al., 1983); en el segundo caso, las cruzas de trigos de invierno x primavera, cuyas progenies seleccionadas en forma alternada en Toluca, Corvallis y Pendeton, han demostrado poseer además de alto rendimiento, estabilidad, fotoinsensibilidad, resistencia a enfermedades y amplia adaptación a ensayos internacionales (Rajaram y Morgounov, 1995) y en el tercer caso, el programa iniciado a partir de 1982 en China ha representado la obtención de líneas de trigo con resistencia a Fusariosis e incrementos y estabilidad en el rendimiento (Rajaram et al., 1983, Rajaram et al., 1994). Para los próximos seis años (2014 - 2020) dos centros internacionales del grupo CGIAR, CIAT y CIMMYT han focalizado sus acciones del mejoramiento genéticos de cultivos mediante la selección multilocal- shuttle breeding- para explorar las características complementarias de diferentes sitios de selección (CIAT, 2014).

Teniendo en cuenta la importancia y los logros del sistema de Shuttle Breeding, se realizó este estudio con el propósito de evaluar el sistema alternado y de determinar entre los sistemas alternados (Toluca-Cd. Obregón, Batán-Cd Obregón y BatánToluca) e in situ (Toluca-Toluca, Cd. ObregónCd. Obregón y Batán-Batán) el que proporciona el mayor número de líneas de trigo con alto rendimiento y estabilidad.

\section{MATERIALES Y MÉTODOS}

Material genético $\mathbf{y}$ avance generacional. Se realizaron seis cruzamientos con diez materiales genéticos de trigo harinero (Vee\#5, Koel, Weaver, Cno79, Seri, Prl, Star, Attila, Roller y Turaco) en las siguientes combinaciones: cruza 1: Vee/ Koel//Weaver; cruza 2: Cno79/Prl//Seri; cruza 
3: Pfau/Vee\#5//Star; cruza 4: Attila/Weaver; cruza 5: Roller/Weaver y cruza 6: Turaco// Pfau/Vee\#5. En cada cruzamiento se obtuvo la generación $\mathrm{F}_{1} \mathrm{y}$ el procedimiento de selección tanto en el sistema alternado como in situ, fue el masal modificado (Rajaram y Morgounov, 1995). La época de siembra en cada localidad para el avance generacional se realizó en Cd. Obregón en el periodo invierno-primavera y en Toluca y Batán en el verano. En la Tabla 1 se presenta la generación, los sistemas de selección y las épocas de siembra por periodo estacional.

\section{Características de las localidades y sistemas de} selección. Las localidades involucradas en el estudio, fueron Cd. Obregón, Toluca y Batán. La primera localidad está situada a 27²9’LN y 10957’LO a una altura de $40 \mathrm{msnm}$, ubicada en el Valle del Yaqui en el Noroeste de México, Estado de Sonora con un tipo de clima designado como $\mathrm{Bw}\left(\mathrm{h}^{\prime}\right) \mathrm{w}\left(\mathrm{e}^{\prime}\right)$. El trigo se cultiva en condiciones de irrigación y en ausencia de enfermedades, excepto la roya de la hoja y del tallo. La siembra se realiza entre los meses de noviembre y diciembre cuando la longitud del día es relativamente corta, con temperaturas bajas. El trigo madura entre los meses de abril y mayo, cuando el día es largo y la temperatura es alta. Este ambiente, considerado subtropical semiárido, presenta los mayores rendimientos y la mejor oportunidad para discriminar genotipos y hacer selección de amplia adaptación (Braun et al., 1996); además, provee la oportunidad de observar la máxima expresión de biomasa y rendimiento (Sayre et al., 1997) y eficiencia en el uso del agua, tolerancia a calor y resistencia a roya de la hoja y del tallo.

La segunda localidad, Toluca en el Estado de México, está situada a 18ำ19’LN y 9939'LO a una altura de 2,640 msnm. El clima es templado húmedo con el invierno benigno $[\mathrm{C}(\mathrm{w})(\mathrm{w}) \mathrm{b}(\mathrm{i}) \mathrm{g}]$; por la alta precipitación y humedad relativa $\mathrm{y}$ temperaturas bajas en la noche se considera un ambiente óptimo para la selección por resistencia a enfermedades como roya amarilla, Septoriosis, BYDV, Fusariosis, además del complejo de enfermedades de la raíz causadas por Helminthosporium sp., Fusarium sp., Pythium sp. y Rizoctonia spp, principalmente (Sayre et al, 1997). La tercera localidad, el Batán está ubicada en el municipio de Texcoco, Estado de México a 1930’LN y 983’은 a una altura de $2.250 \mathrm{msnm}$. El clima es templado semi-seco. Por las características de clima, principalmente la temperatura media de $19,5^{\circ} \mathrm{C}$ se considera un ambiente propicio para la selección por resistencia a roya del tallo y el desarrollo del trigo en condiciones de temporal.

Tabla 1. Generaciones y sistemas de selección alternada e in situ en tres localidades en México.

\begin{tabular}{llllllll}
\hline \multirow{2}{*}{ Generación } & \multicolumn{7}{c}{ Sistema de selección } \\
& T-Y & B-Y & B-T & T-T & Y-Y & B-B & \\
\hline $\mathrm{F}_{2}$ & - & - & - & $\mathrm{T}-02$ & $\mathrm{Y}-01 / 02$ & $\mathrm{~B}-02$ \\
$\mathrm{~F}_{3}$ & $\mathrm{Y}-02 / 03$ & $\mathrm{Y}-02 / 03$ & $\mathrm{~T}-03$ & $\mathrm{~T}-03$ & $\mathrm{Y}-02 / 03$ & $\mathrm{~B}-03$ \\
$\mathrm{~F}_{4}$ & $\mathrm{~T}-03$ & $\mathrm{~B}-03$ & $\mathrm{~B}-04$ & $\mathrm{~T}-04$ & $\mathrm{Y}-03 / 04$ & $\mathrm{~B}-04$ \\
$\mathrm{~F}_{5}$ & $\mathrm{Y}-03 / 04$ & $\mathrm{Y}-03 / 04$ & $\mathrm{~T}-05$ & $\mathrm{~T}-05$ & $\mathrm{Y}-04 / 05$ & $\mathrm{~B}-05$ \\
\hline
\end{tabular}

T= Toluca (verano); Y=Cd. Obregón (invierno-primavera) y B=Batán (verano); 02-03-04-05= años 2002, 2003, 2004 y 2005 , respectivamente. 
Método de selección. Se utilizó el método de selección masal modificado (Rajaram et al., 1983; Rajaram et al., 1994) descrito así: $\mathrm{F}_{1}$ : en Toluca en el ciclo de verano se realizaron los cruzamientos involucrando los diez progenitores de trigo descritos anteriormente; $\mathrm{F}_{2}$ : en Cd Obregón en el ciclo invierno-primavera se sembraron 2000 plantas espaciadas por cruza; se aplicó una presión de inoculo natural de roya de la hoja y del tallo y se realizó la selección individual con base en tipo agronómico, resistencia a enfermedades y tipo de semilla; $\mathrm{F}_{3}$ : en Toluca en el ciclo de verano se sembró la semilla de cada planta individual $\mathrm{F}_{2}$ para producir plantas $\mathrm{F}_{3}$ a una densidad de siembra comercial de $100 \mathrm{~kg} / \mathrm{ha}$ en tres surcos de dos metros por línea. La selección se basó en tres características: biomasa, densidad de espigas y resistencia a enfermedades entre líneas y no dentro de líneas. Dentro de las líneas seleccionadas se tomaron de 10 a 15 espigas por línea, las cuales fueron trilladas; al final de este paso se realizó la selección por tipo de grano. Las líneas seleccionadas conformaron la generación $\mathrm{F}_{4}$ que fueron sembradas en Cd. Obregón en el ciclo invierno-primavera en alta densidad y la selección se realizó por resistencia múltiple a enfermedades y biomasa; $\mathrm{F}_{5}$ : en Toluca de igual forma que en $\mathrm{F}_{3}$ o $\mathrm{F}_{4}$. Los métodos estudiados fueron: Alternados (Toluca-Cd. Obregón, Batán-Cd Obregón y BatánToluca) denominados con la abreviatura T-Y, B-Y y B-T, respetivamente e in situ (Toluca-Toluca, Cd. Obregón-Cd. Obregón y Batán-Batán) denominados con la abreviatura T-T, Y-Y y B-B, respectivamente (Tabla 1).

Evaluación de los sistemas de selección (EESS) y diseño experimental. Al final del proceso de la selección masal modificada, en la generación $\mathrm{F}_{5}$ se seleccionaron 32 líneas por cruza y sistema de selección, para un total de 192 líneas. Se utilizaron 4 variedades testigos para conformar un EESS ajustado a un diseño alfa-lattice 14 x 14 con tres repeticiones. Las variedades testigos fueron: Bacanora T88, Weaver, Tui y Specht. La unidad experimental se constituyó de dos surcos dobles de $3.0 \mathrm{~m}$ de longitud para un área de cosecha útil de $4,5 \mathrm{~m}^{2}$.

Siembra y manejo agronómico. La siembra y cosecha en cada localidad se realizaron en Cd Obregón (26/11/2005-08/05/2006),enBatán(06/06/2006$06 / 10 / 2006)$ y en Toluca (01/06/2006). La densidad de siembra fue de $100 \mathrm{~kg} / \mathrm{ha}$, depositando 270 semillas $/ \mathrm{m}^{2}$. La siembra y cosecha en las tres localidades se realizó con sembradora y cosechadora mecánica. El manejo agronómico de los EESS en relación con la nutrición se realizó aplicando NPK en Cd Obregón (150-46-60) y en Batán y Toluca (15046-00). No se realizaron control para patógenos con el fin de evaluar la resistencia en condiciones de inoculo natural.

Variables evaluadas y análisis estadístico. En la época de cosecha, en cada localidad, se determinó el rendimiento de grano mediante la cosecha de la unidad experimental útil de $4,5 \mathrm{~m}^{2}$, expresada en $\mathrm{kg} / \mathrm{ha}$ y ajustada a una humedad del $14 \%$. En el ensayo de líneas y en los cuatro testigos, además del rendimiento, biomasa, índice de cosecha, granos $/ \mathrm{m}^{2}$ y peso de mil granos, se realizaron los siguientes procedimientos estadísticos: 1) Análisis individual de Varianza por localidad, para determinar la variabilidad entre los genotipos; 2) prueba de homogeneidad de varianzas del error en cada una de las localidades según la Prueba de Bartlett; 3) comparación de medias mediante la prueba de Tukey $(\mathrm{p}=0,05)$. Se realizó, solo para los testigos utilizados, el análisis combinado de localidades, con el modelo estadístico que correspondió a:

$$
Y_{i j k}=\mu+G_{i}+A_{j}+(G A)_{i j}+B_{k(j)}+E_{i j k}
$$

Donde $Y=$ variable de estudio; $\mu$ = efecto de la media, $G_{i}=$ tratamiento (líneas de trigo); $A_{j}=$ ambientes (3 localidades); $(\mathrm{GA})_{\mathrm{ij}}=$ interacción genotipo $\mathrm{x}$ ambiente; $\mathrm{B}_{\mathrm{k}(\mathrm{i})=}$ repeticiones (3) y $\mathrm{E}_{\mathrm{ijk}}=$ error experimental. 
El análisis de la interacción genotipo por ambiente, se hizo con base en el método de estabilidad fenotípica de Eberhart y Russell (1966). Para el Análisis de Varianza, las localidades y las repeticiones se consideran efectos aleatorios y los genotipos, efectos fijos. La estabilidad de cada genotipo se describió por parámetros definidos en el siguiente modelo matemático:

$$
Y_{i j}=X_{i j}+\beta_{i} X_{j}+d_{i j}+e_{i j} \text {; }
$$

Dónde: $Y_{\mathrm{ij}}$ : promedio del i-ésimo tratamiento en el j-ésimo ambiente; $X_{\mathrm{ij}}$; promedio del iésimo tratamiento sobre todos los ambientes; $\beta_{i}$ : coeficiente de regresión lineal que mide la respuesta del i-ésimo tratamiento en los diferentes ambientes; $\mathrm{X}_{\mathrm{j}}$ : índice del j-ésimo ambiente; $d_{i j}$; desviación a partir de la línea de regresión correspondiente al iésimo tratamiento en el j-ésimo ambiente y $\mathrm{e}_{\mathrm{ij}}$ : error asociado al i-ésimo tratamiento en el j-ésimo ambiente según el modelo lineal aditivo.

\section{RESULTADOS Y DISCUSIÓN}

Evaluación y selección del mejor testigo. La identificación del mejor testigo, se realizó con el fin de determinar el número y frecuencias de líneas superiores a él en cada uno de los seis sistemas de selección. El Análisis de Varianza combinado de las localidades Cd. Obregón, Batán y Toluca solo para los cuatro testigos presentó diferencias altamente significativas para los factores localidad (L), testigo (T) y la interacción T x L para todas las variables estudiadas (R, B, IC, EM2 y PMG) con excepción de biomasa (B) que no presentó diferencias estadísticas para el factor testigo y la interacción T x L (Tabla 2). No se presentaron diferencias estadísticas entre los testigos Bacanora T88 y Spech con excepción del peso de mil granos, donde Spech $(30,20 \mathrm{~g})$ fue diferente estadísticamente con relación a Bacanora T88 (26,46 g). Tampoco se observaron diferencias estadísticas para las variables R, B, IC y GM2 entre los testigos Spech y Bacanora T88. Sin embargo, se seleccionó para efectos del estudio a Spech como el punto de comparación de las líneas seleccionadas de cada sistema (Tabla 3).

\section{Evaluación de líneas por sistema de selección.} El ANDEVA para el rendimiento de grano, presentó diferencias altamente significativas entre localidades (L), sistemas (S) y la interacción localidades por sistemas (LxS) (Tabla 4). Las diferencias estadísticas entre localidades, marcaron las grandes diferencias ecológicas entre ellas. Además, en el contexto de los objetivos planteados, son importantes las diferencias detectadas para la interacción LxS, ya que se requiere que esta interacción sea estadísticamente significativa para proceder a estimar y describir sus componentes (Sprague y Federer, 1951; Plaisted y Peterson, 1959).

Tabla 2. Cuadrados medios y coeficientes de variación (CV) para el rendimiento de grano (R), biomasa (B), índice de cosecha (IC), granos $/ \mathrm{m}^{2}\left(\mathrm{GM}^{2}\right)$ y peso de mil granos (PMG) de cuatro variedades testigos de trigo evaluadas en Cd. Obregón, Batán y Toluca, México.

\begin{tabular}{lllllll}
\hline FV & GL & \multicolumn{1}{c}{$\mathbf{R}$} & \multicolumn{1}{c}{ B } & \multicolumn{1}{c}{ IC } & \multicolumn{1}{c}{$\mathbf{G M}^{2}$} & \multicolumn{1}{c}{ PMG } \\
\hline Localidad (L) & 2 & $43,48^{* *}$ & $1268,03^{* *}$ & $0,0295^{* *}$ & $276,685^{* *}$ & $146,55^{* *}$ \\
Rep/Localidades & 6 & 0,11 & 26,30 & 0,0021 & 1,800 & 1,28 \\
Testigos (T) & 3 & $2,21^{* *}$ & 31,645 & $0,0097^{* *}$ & $15,36^{* *}$ & $85,10^{* *}$ \\
T x L & 6 & $1,32^{* *}$ & 43,002 & $0,0031^{* *}$ & $12,01^{* *}$ & $14,92^{* *}$ \\
\hline Error & 18 & 0,13 & 23,063 & 0,0010 & 2.779 & 2,94 \\
CV & & 8,26 & 14,74 & 7,43 & 10,72 & 1,71 \\
\hline
\end{tabular}


Tabla 3. Medias y coeficientes de regresión para el rendimiento de grano (R), biomasa (B), índice de cosecha (IC), granos $/ \mathrm{m}^{2}$ (GM2) y peso de mil granos (PMG) de cuatro variedades testigos de trigo evaluadas en Cd. Obregón, Batán y Toluca, México.

\begin{tabular}{lccccc}
\hline Variedad & $\begin{array}{c}\mathbf{R} \\
\mathbf{t} / \mathbf{h a}\end{array}$ & $\begin{array}{c}\mathbf{B} \\
\mathbf{t} / \mathbf{h a}\end{array}$ & $\begin{array}{c}\text { IC } \\
\text { número }\end{array}$ & $\begin{array}{c}\text { GM2 } \\
\text { número }\end{array}$ & $\begin{array}{c}\text { PMG } \\
\mathbf{g}\end{array}$ \\
\hline \multirow{2}{*}{ Bacanora T88 } & $4,76 \mathrm{a}$ & $10,4 \mathrm{a}$ & $0,451 \mathrm{a}$ & $17.099 \mathrm{a}$ & $27,46 \mathrm{~b}$ \\
& $(0,90)$ & $(0,94)$ & $(0,57)$ & $(1,03)$ & $(0,66)$ \\
Weaver & $3,80 \mathrm{~b}$ & $9,4 \mathrm{a}$ & $0,388 \mathrm{a}$ & $14,740 \mathrm{~b}$ & $24,39 \mathrm{c}$ \\
& $(1,21)$ & $(1,16)$ & $\left(1,46^{* *}\right)$ & $(1,57 * *)$ & $(0,86)$ \\
Tui & $4,56 \mathrm{a}$ & $10,5 \mathrm{a}$ & $0,427 \mathrm{a}$ & $12.740 \mathrm{~b}$ & $31,26 \mathrm{a}$ \\
& $(0,97)$ & $(0,99)$ & $(0,79)$ & $(0,96)$ & $(0,86)$ \\
Spech & $4,95 \mathrm{a}$ & $10,8 \mathrm{a}$ & $0,451 \mathrm{a}$ & $16,140 \mathrm{ab}$ & $30,20 \mathrm{a}$ \\
Promedio & $(0,97)$ & $(0,96)$ & $(0,89)$ & $(0,92)$ & $(0,92)$ \\
DMS $(0,05)$ & 4,52 & 10,3 & 0,429 & 15,054 & 28,33 \\
\hline
\end{tabular}

Medias con la misma letra son estadísticamente iguales al 0,05\% de probabilidad. ${ }^{* *}$, Significancia del coeficiente de regresión (entre parantesis) diferente a la unidad al 1\% de probabilidad.

Tabla 4. Cuadrado medio y coeficiente de variación (CV) para el rendimiento de grano de seis sistemas de selección evaluados en Cd.

Obregón, Batán y Toluca, México

\begin{tabular}{lll}
\hline FV & GL & Rendimiento de grano \\
\hline Localidad (L) & 2 & $83,53^{* *}$ \\
Rep/Localidad & 6 & 0,26 \\
Sistemas (S) & 5 & $0,29^{* *}$ \\
S x L & 10 & $0,30^{* *}$ \\
\hline Error & 30 & 0,01 \\
CV (\%) & & 7,71 \\
\hline
\end{tabular}

**= Significativo al $1 \%$ de probabilidad.

En la Tabla 5 se presenta la media, el coeficiente de regresión y el cuadrado medio de las desviaciones de regresión del rendimiento de grano para cada uno de los sistemas de selección evaluados. Adicionalmente, se presenta el número y porcentaje de líneas superiores y estables con relación al promedio general (sistemas más testigos) y con relación al testigo (Spech). Los resultados mostraron que el mayor rendimiento se obtuvo con el sistema alternado B-T con 4,7 t/ha, el cual superó estadísticamente al sistema in situ B-B y Y-Y cuyo rendimiento fue de 4,60 y 4,55 t/ha, 
respectivamente y los sistemas BB y T-Y fueron iguales estadísticamente entre sí. El sistema B-T superó en 160 y $240 \mathrm{~kg}$ a los sistemas B-B y T-Y que ocuparon el segundo y tercer lugar, respectivamente. Los sistemas B-Y $(4,37 \mathrm{t} / \mathrm{ha}) \mathrm{y}$ T-T $(4,30 \mathrm{t} / \mathrm{ha})$ presentaron el menor rendimiento y difirieron estadísticamente en 390 y $460 \mathrm{~kg}$ menos del sistema de mayor rendimiento (B-T).

Los coeficientes de regresión (bi) de cada uno de los sistemas de selección fueron estadísticamente iguales a la unidad; sin embargo, el sistema B-T presentó el coeficiente de regresión numéricamente más cercano a la unidad $(0,99)$; además, este sistema proporcionó el mayor número y porcentaje de líneas $(22,0$ y 11,5\%) con rendimientos superiores y estables al promedio general (4,52 t/ha). Así mismo, con relación al testigo
(Spech: 4,95 t/ha) este sistema presentó el mayor número y porcentaje de líneas superiores y estables $(12,0$ y $6,3 \%)$.

El sistema de selección in situ B-B presentó el segundo coeficiente de regresión más cercano a la unidad $(1,06)$ aportando 18 líneas con rendimiento superior a la media general, de los cuales 16 fueron estables; 8 líneas superaron al testigo Spech y además fueron estables. El tercer lugar, lo ocupo el sistema T-Y que no obstante de presentar el coeficiente de regresión más distante a la unidad de los seis sistemas, presentó un número de líneas con alto rendimiento y estables con relación a la media general igual al aportado por el sistema B-B. Los sistemas B-Y y T-T presentaron los menores rendimientos y aportaron el menor número de líneas de alto rendimiento y estables.

Tabla 5. Estimaciones de la media y parámetros de estabilidad para el rendimiento de grano $(\mathrm{t} / \mathrm{ha})$, número y porcentaje de líneas superiores en cada sistema de selección de trigo.

\begin{tabular}{|c|c|c|c|c|c|c|c|}
\hline \multirow{3}{*}{ Sistema } & \multirow{2}{*}{\multicolumn{3}{|c|}{ Parámetro }} & \multicolumn{4}{|c|}{ Número y líneas superiores (\%) respecto a: } \\
\hline & & & & Promedio & general & Testigo & \\
\hline & $\mathbf{U}_{\mathbf{i}}$ & $\mathbf{b}_{\mathrm{i}}$ & $\mathbf{S}^{2} \mathbf{d}_{\mathrm{ij}}$ & Superior & Estable & Superior & Estable \\
\hline \multicolumn{8}{|l|}{ Alternado } \\
\hline $\mathrm{T}-\mathrm{Y}$ & $4,55 \mathrm{~b}$ & 1,14 & 0,037 & $18(9,4)$ & $16(8,3)$ & $5(2,6)$ & $4(2,1)$ \\
\hline B-Y & 4,37 de & 1,09 & 0,007 & $12(6,3)$ & $9(4,7)$ & $4(2,1)$ & $3(1,6)$ \\
\hline B-T & $4,76 \mathrm{a}$ & 0,99 & 0,009 & $22(11,5)$ & $22(11,5)$ & $12(6,3)$ & $12(6,3)$ \\
\hline \multicolumn{8}{|l|}{ in situ } \\
\hline $\mathrm{T}-\mathrm{T}$ & $4,30 \mathrm{e}$ & 0,90 & 0,044 & $12(6,3)$ & $12(6,3)$ & $5(2,6)$ & $5(2,6)$ \\
\hline Y-Y & $4,47 \mathrm{~cd}$ & 1,09 & $0,471^{* *}$ & $17(8,9)$ & $17(8,9)$ & $5(2,6)$ & $5(2,6)$ \\
\hline B-B & $4,60 \mathrm{~b}$ & 1,06 & 0,003 & $18(9,4)$ & $16(8,3)$ & $8(4,2)$ & $8(4,2)$ \\
\hline
\end{tabular}

$\mathrm{DMH}=$ diferencia mínima honesta $(0,05)$ entre sistemas $=0,1186$. Medias con la misma letra son iguales estadísticamente (Tukey 0,05\%). * **= diferencias al 5 y $1 \%$ de 1 para $b_{\mathrm{i}}$ y de cero para $\mathrm{S}^{2} \mathrm{~d}_{\mathrm{ij}}$. Entre paréntesis, porcentaje de líneas superiores. 
La idea generalizada de que la selección a través de localidades es más efectiva que la selección en un solo sitio específico para mejorar estabilidad, aquí no resulto consistente. Para este estudio, tanto el sistema de selección alternada B-T como el in situ B-B, presentaron rendimiento alto y estable. En trigo, resultados presentados por Crossa et al. (1991) sugieren y apoyan este resultado. Una de las hipótesis de este estudio, se basó en suponer que los sistemas de selección alternados aportan genotipos más estables que los in situ. En la comparación de los dos grupos de sistemas tomando el rendimiento promedio de los tres sistemas alternados y el de los tres in situ, la selección alternada con relación a la in situ resulto superior en 2,41\% en rendimiento (promedio selección alternada de 4,56 t/ha vs promedio selección in situ de 4,45 t/ha), un rendimiento superior de solo $110 \mathrm{~kg}$.

No obstante, el éxito reconocido del sistema tradicional de selección alternada T-Y iniciado por Borlaug (1968) en México, los resultados del presente estudio indican que este sistema no es el mejor sino el sistema B-T en relación con el número de líneas de alto rendimiento y estables. El hecho de que el sistema B-T haya resultado aquí el mejor, podría atribuirse a las siguientes causas; a) las características del material genético estudiado y b) que realmente podrían existir otras opciones de sistemas alternados para obtener alto rendimiento y estabilidad. En relación con la primera causa, las seis cruzas y sus progenitores que dieron origen a los seis sistemas de selección, fueron el producto del sistema alternado T-Y, el cual ocasionó que el comportamiento de los sistemas alternados e in situ no fueran independientes del origen del material genético de partida, al llevar incorporado el efecto de la selección bajo el sistema tradicional T-Y.

Al respecto, Young y Frey (2004) mencionan que el éxito de un programa de selección alternada, depende tanto de la base genética del material usado, como de las características y poder discriminante de los ambientes involucrados. Supuestamente, el programa iniciado por Borlaug (1968) presenta estas dos características mencionadas por estos dos autores. La correlación entre la media del rendimiento y el coeficiente de regresión $\left(\mathrm{b}_{\mathrm{i}}\right)$ fue baja y no significativa en los sistemas B-T y B-B ( $r=-$ 0,06 y $r=-0,15$, respectivamente). La ausencia de correlación es un indicativo de que B-T y B-B son los sistemas más estables, a diferencia de los sistemas T-Y, B-Y y Y-Y cuyas medias de rendimiento y coeficientes de regresión presentaron una correlación significativa y positiva $\left(\mathrm{r}=0,40^{* *}, \mathrm{r}=0,59^{* *} \mathrm{y}\right.$ $r=0,57^{* *}$, respectivamente), mientras que el sistema T-T tuvo una correlación $r=-0,60^{* *}$. La presencia de esta correlación positiva, indica adaptación específica. Estos resultados concuerdan con los obtenidos por Perry y D’Antuono (1989) y Trethowan et al. (2007) quienes encontraron que la selección de genotipos de trigo para alto rendimiento, se asocia con adaptación específica. De igual forma, la asociación negativa entre las medias del rendimiento y los coeficientes de correlación de las líneas proporcionadas por el sistema in situ T-T era de esperarse; es la respuesta opuesta a la de los sistemas T-Y, B-Y y Y-Y con adaptación específica a ambientes óptimos. En el caso del sistema T-T, se presentó adaptación específica de las líneas pero a ambientes limitativos.

Estos resultados indican que las líneas de alto rendimiento proporcionadas por los sistemas T-Y, B-Y y Y-Y presentan una alta relación con ambientes óptimos, en este caso el de Cd. Obregón, como ambiente de selección en común. Esto corrobora y está directamente relacionado con la clasificación que tiene el CIMMYT de los mega ambientes donde el objetivo del sistema T-Y es proporcionar líneas de alto rendimiento para el ME1, definido como óptimo. Este megaambiente (ME1) representa el $40 \%$ del área de siembra en países en desarrollo con 32 millones de hectáreas y provee variedades con las siguientes características: a) variedades de porte bajo (Rht1 y Rht2); b) fotoinsensibles (Ppd1 o Ppd2); c) alto potencial de rendimiento con re- 
gistros experimentales de rendimiento hasta de 10,0 t/ha; d) respuesta y eficiencia a insumos; e) resistencia a la roya del tallo basado en el complejo Sr2 y f) tolerancia al calor y volcamiento (Rajaram y Morgounov, 1995).

\section{CONCLUSIONES}

El mejor sistema fue B-T por haber presentado el mayor rendimiento promedio diferente significativamente a los demás sistemas y el mayor número de líneas de alto rendimiento y estables. En contraposición, los sistemas B-Y, T-T y Y-Y fueron los que aportaron el menor número de líneas a alto rendimiento y estables.

\section{REFERENCIAS BIBLIOGRÁFICAS}

ADEGOKE, A. 0. y FREY, K. J. 1981. Production traits of oat strains from disruptively and non-disruptively propagated bulk populations in oats, Egyptian journal of genetics and cytology. 10:199 - 212.

BYERLEE, D. y MOYA, P. 1993. Impacts of international wheat breeding research in the developing world, 1969-90. Mexico, D.F. CIMMYT. 135 p.

BRAUN, H. J., RAJARAM, S. y VAN GINKEL, M. 1996. CIMMYT's approach to breeding wheat for wide adaptation. Euphytica. 92:175 - 183

BORLAUG, N. E. 1968. Wheat, rust, and people. Phytopathology. 55:1088 - 1098.

CENTRO INTERNACIONAL DE AGRICULTURA TROPICAL (CIAT). 2014. Estrategia CIAT 2014-2020. Construyendo un futuro ecoeficiente. CIAT, Cali, Colombia. 48 p.

CROSSA, J., FOX, P. N., PFEIFFER, W., RAJARAM, S. y GAUCH, H. G. 1991. AMMI adjustment for statistical analysis of two international maize cultivar trials. Theoretical and Applied Genetics. 81:27 - 37.

EBERHART, S. A. y RUSSELL, W. A. 1966. Stability parameters for comparing varieties. Crop Science. 6:36 - 40.

LANTICA, M.A., DUBIN, H.J y MORRIS, M.L. 2005 Impacts of International Wheat Breeding Research in the Developing World, 1988-2002. México, DF: CIMMYT.

LU Y. C., TSAI K. H. y OKA I. 1967. Studies of soybean breeding in Taiwan. 1. Breeding experiments with successive hybrid generations grown in different season. Botanical Bulletin of Academia Sinica. 8:80 - 90.
PERRY, M. W. y D'ANTUONO, M. E. 1989. Yield improvement and associate characteristics of some Australian spring wheats cultivars between 1860 and 1982. Australian Journal of Agricultural Research. 40:457 - 472.

PLAISTED, R. L. y PETERSON, L. C. 1959. A technique for evaluating the ability of selections to yield consistently in different locations and seasons. American Potato Journal. 36:381 - 385

RAJARAM, S., MANN, CH., ORTIZ-FERRARA, G. y MUJEEBKAZI, A. 1983. Adaptation, stability and high yield potential of certain IB/IR CIMMYT Wheats. Proc. Int. Wheat Genet. Symp. 6 th. $613-621$.

RAJARAM, S., GINKEL, M. y FISCHER, R. A. 1994. CIMMYT's wheat breeding Mega-environments (ME). In Proceedings of the 8th International Wheat Genetic Symposium. Beijing, China. $1101-1106 \mathrm{p}$.

RAJARAM, S. y MORGOUNOV, I. 1995. Wheat Germplasm Improvement at CIMMYT Mexico. Proc. Of the Ukaraine/ CIMMYT Workshop. In: Heat Program Special Report WPSR No $37.120 \mathrm{p}$.

REYNOLDS, M. P., PASK, A. S. D., MULLAN, D. M. y CHAVEZDULANTO, P. N. 2013. Fitomejoramiento fisiológico I: Enfoque interdisciplinario para mejorar la adaptación del cultivo. México, D.F: Centro Internacional de Mejoramiento de Maíz y Trigo (CIMMYT). $174 \mathrm{p}$.

REYNOLDS, M. P. y BORLAUG, N. E. 2006. Impacts of breeding on international collaborative wheat improvement. Journal of Agricultural Science. 144:3 - 1.

SAYRE, K. D., RAJARAM, S. y FISHER, R. A. 1997. Yield Potential progress in short Bread Wheat's in Northwest Mexico. Crop Science. 37:36 - 42.

SHABANA, R T., BAILEY, T. y FREY, K. J. 1980. Production traits of oats selected under low, medium and high productivity. Crop Science. 20:739 - 744.

SPRAGUE, G. F. y FEREDER, W. I. 1951. A comparison of variance component in corn yield trials. Agronomy Journal. $43: 535$ - 541.

TRETHOWAN, R.M., REYNOLDS, M.P., ORTIZ-MONASTERIO, J.I. y ORTIZ, R. 2007. The genetic basis of the green revolution on in wheat production. Plant Breeding Reviews. 28:39 - 58.

YOUNG, C. y FREY, K. J. 1994. Grain-yield characteristics of oat lines surviving uniform and shuttle strategies. Euphytica. 76, $63-71$ 